\title{
STRUCTURAL CHARACTERISTICS OF SPIRAL BULGES
}

\author{
YONG-IK BYUN \\ Institute of Astronomy, National Central University \\ Chung-Li, 32054, Taiwan
}

\section{Introduction}

Recently it has been suggested that some bulges are really disks (Kormendy 1993). He argues that some bulges are not spheroidal components of steep luminosity distribution like elliptical galaxies but actually parts of flat disks, whose central luminosity profiles are steeper than the inward extrapolation of an exponential fit to the outer parts. The existence of disk-like bulges strongly supports the idea of disk secular evolution.

Based on a large sample of spiral galaxy images, I examine below structural characteristics of bulge components in more direct way. This work is based on the I-band CCD image library of 1355 southern spiral galaxies (Sb and later Hubble type, diameter larger than 1.5 arcmin) provided by Prof. Mathewson (Mathewson, Ford, and Buchhorn 1992). A two-dimensional decomposition procedure, an improved version of the method described in Byun and Freeman (1995), was applied to images to extract structural parameters of bulge and disk components. scalelength parameters, and shape parameters for bulge and disk. Since this work is focused on the bulge component, galaxies with weak or no bulges $(B / D<0.05)$ were removed from the sample. Galaxies classified as barred spirals in ESO catalogue were also removed because the presence of a strong bar would badly contaminate bulge parameters in the present decomposition scheme. Final sample consists of a total of 491 galaxies.

\section{True Flattening, Scalelength and Luminosity of Bulges}

Since the viewing inclination of galaxies can be estimated from their disk axial ratios, with an assumed value of true disk axial ratio, the true flattening of bulge component can be derived in a straightforward manner. The inspection of the distribution of intrinsic bulge axial ratio proves that many 
bulges are close to spherical. They are also more easily seen at lower inclinations probably because it becomes more difficult to observe small bulges at high inclinations. At high inclinations $\left(i>70^{\circ}\right)$, there appears to be an excess of somewhat flattened bulges $(c / a \sim 0.7)$; this excess may correspond to boxy/peanuts bulges frequently found in edge-on galaxies. No such excess can be found in the low inclination group $\left(i<70^{\circ}\right)$. Bulge axial ratios and their distribution appear to have no particular dependence on Hubble types nor on total luminosity. Their true axial ratio ranges from 0.4 to 1.0 with peak at 1.0 for both $\mathrm{Sc}$ and $\mathrm{Sb}+\mathrm{Sbc}$ galaxies. Without velocity dispersion information, I cannot tell if flattened bulges are systematically colder than spherical bulges.

The bulge and disk scalelength parameters, converted to metric scale assuming an uniform Hubble flow, show a positive correlation but with significant scatter. Similar correlation was found by Courteau, de Jong, and Broeils (1996) and used as an evidence for bulges being product of disk secular evolution because their slope of 0.08 was close to the ratio of bulge to disk scalelengths of 0.1 predicted by simulations. But my scalelength correlation does not provide strong support for the secular evolution scenario of bulge formation; it has much smaller slope and large scatter.

Bulges are on average about 2 magnitudes fainter than disks and luminous disks have general tendency to possess luminous bulges. But this correlation is also with a big scatter, which corresponds to a range of $B / D$ values. A few galaxies have bulges almost as luminous as disks while others have much fainter bulges at a given disk luminosity. Sc galaxies have generally fainter bulges than $\mathrm{Sb}$ galaxies, but the scatter in the correlation is also as large as in $\mathrm{Sb}$.

There is a general tendency that bigger luminous disks have bigger luminous bulges; but could it be that bigger proto-galaxies have evolved into bigger disks and bulges? or that bigger bulges have led bigger disk formation? The scatter may be a sign that bulge and disk formation is not simple and more than one process is responsible.

I am grateful to Professor D. Mathewson, who made his CCD images available for my study. This work was supported by the National Science Council of Republic of China through the grant NSC 87-2112-M-008-025.

\section{References}

Byun, Y. I. \& Freeman, K. C. 1995, ApJ, 448, 563

Courteau, S., de Jong, R. S., \& Broeils, A. H. 1996, ApJ, 457, L73

Kormendy, J. 1993, in Galactic Bulges, ed. H. Dejonghe \& H. J. Habing (Dordrecht: Kluwer), 209

Mathewson, D. S., Ford, V. L., \& Buchhorn, M. 1992, ApJS, 81, 413 